$16^{\circ}$ USIHC - Congresso Internacional de Ergonomia e Usabilidade de USIHC Interfaces Humano Computador

CINAHPA

CINAHPA | 2017 - Congresso Internacional de Ambientes Hipermídia para Aprendizagem.

\title{
MEDINDO A EXPERIÊNCIA DO USUÁRIO POR MEIO DE SINAIS FISIOLÓGICOS
}

\section{MEASURING THE USER EXPERIENCE THROUGH PHYSIOLOGICAL SIGNS}

\author{
Tiago Catecati ${ }^{1}$, M.Sc. \\ Eugenio Andrés Díaz Merino², D.Sc. \\ Bruno Maia de Guimarães ${ }^{3}$, D.Sc., \\ Aline Girardi Gobbi ${ }^{4}$, M.Sc. \\ Marcelo Gitirana Gomes Ferreira ${ }^{5}$, D.Sc. \\ (1) Universidade Federal de Santa Catarina \\ e-mail: tcatecati@gmail.com \\ (2) Universidade Federal de Santa Catarina \\ e-mail: eugenio.merino@ufsc.br \\ (3) Universidade Federal de Santa Catarina \\ e-mail:bmguimaraes@hotmail.com \\ (4) Universidade Federal de Santa Catarina \\ e-mail: aline.gobbi@gmail.com \\ (5) Universidade do Estado de Santa Catarina \\ e-mail: marcelo.gitirana@gmail.com
}

Palavras-chave: Satisfação do usuário, sinais fisiológicos, usabilidade.

Esta pesquisa teve como objetivo identificar os principais equipamentos utilizados na medição de sinais fisiológicos, passíveis de serem empregados na avaliação da satisfação do usuário em testes de usabilidade. Foi desenvolvida uma revisão bibliográfica sistemática para identificar as dez tecnologias de medição de sinais fisiológicos abordadas neste artigo.

Key-words: User satisfaction, physiological signals, usability.

This research aimed to identify the main equipment used in the measurement of physiological signals, which can be used to evaluate user satisfaction in usability tests. A systematic literature review was developed to identify the ten physiological signal measurement technologies discussed in this article. 
$16^{\circ}$ Ergodesign - Congresso Internacional de Ergonomia e Usabilidade de Interfaces Humano Tecnológica: Produto, Informações Ambientes Construídos e Transporte

$16^{\circ}$ USIHC - Congresso Internacional de Ergonomia e Usabilidade de Interfaces Humano Computador

CINAHPA | 2017 - Congresso Internacional de Ambientes Hipermídia para Aprendizagem.

\section{INTRODUÇÃO}

A experiência do usuário (UX) pode ser entendida como uma extensão do conceito de usabilidade, sendo a usabilidade mais objetiva em relação a interação usuário e produto.A UX considera os aspectos da usabilidade, mas tem ênfase na análise da satisfaçãodo usuário em relação ao produto. (ARAUJO, 2014)

A usabilidade é definida, pela ABNT (2002), como o conjunto da eficácia, da eficiência e da satisfação, onde é avaliado, em um contexto específico, se usuários específicos conseguem atingir objetivos específicos.

Segundo Jordan (1998), é de costume ser adotados métodos e técnicas tanto empíricos quanto não empíricos, para a avaliação da usabilidade. O que também se aplica a UX. Para as avaliações não empíricas podem ser utilizadas técnicas como as avaliações heurísticas (NIELSEN, 1993) e percursos cognitivos (WHARTON, et al., 1994), com o objetivo de adequar o sistema para um conjunto pré-estabelecido de critérios. Estas avaliações são realizadas por especialistas e geralmente antes do lançamento do produto no mercado, sendo direcionadas para a descoberta de problemas na interface do sistema avaliado.

Nas avaliações empíricas são analisados aspectos da usabilidade através de usuários reais que interagem diretamente com o sistema, produto, ou com um protótipo que possibilite avaliar os aspectos estudados. Rubin \& Chisnell (2008, p. 21) destacam os experimentos controlados com usuários, dentre os métodos empíricos para a avaliação da usabilidade. Os experimentos são embasados na metodologia clássica e são passíveis de serem reproduzidos. Porém os autores expõem que geralmente os testes utiliza abordagens informais e de natureza qualitativa, sem comprometer sua utilidade para o desenvolvimento de sistemas e produtos.

Para o desenvolvimento dos experimentos são planejadas tarefas para serem executadas pelos usuários durante a interação com o sistema ou produto estudado. Durante os testes são avaliados os aspectos de eficiência, eficácia e satisfação do usuário enquanto ele completa as tarefas previstas. Para dar suporte a estas avaliações, são usadas tecnologias como câmeras de vídeo para avaliar aspectos como a forma de interação do usuário com o sistema ou produto; microfones que mantem o registro do que é expresso pelo usuário, principalmente em métodos como o think aloud onde o usuário relata verbalmente todas as ações que desempenha durante o teste (HERTZUM, 2013); cronômetros para marcar o tempo de execução de cada tarefa; softwares que gerenciam estas tarefas de monitoramento; e questionários aplicados logo após a execução das tarefas, que auxiliam na identificação de elementos subjetivos relacionados à satisfação do usuário.

Além dos dados obtidos através dos métodos e tecnologias discutidos, os participantes dos testes de usabilidade geram outras informações como linguagem corporal, onde é possível observar a inquietação do usuário pela maneira que ele se mexe durante o teste ou mesmo as expressões faciais que podem dar indícios de frustração ou outro tipo de emoção. Mesmo que a maior parte desta linguagem corporal seja notada pelos observadores na hora do teste ou por meio das filmagens realizadas durante os testes, alguns comportamentos são mais difíceis de observar por esses meios ou mesmo ainda são imperceptíveis a nível visual. Alguns exemplos são: Rápidas contrações faciais; Dilatação da pupila; Alterações na frequência cardíaca e Alteração da transpiração. Para captar os dados provenientes destas alterações são exigidos equipamentos de medição especializados (ALBERT \& TULLIS, 2013).

Este estudo tem como objetivos identificar os principais equipamentos de medição fisiológica, passíveis de serem utilizados em testes de usabilidade, e abordar os aspectos gerais de cada uma destas tecnologias. Para coletar estas informações de maneira objetiva foi desenvolvida uma revisão bibliográfica sistemática com o objetivo de identificar os principais trabalhos acadêmicos que descrevem a utilização destes equipamentos em conjunto com testes de usabilidade ou com o intuito que associar a 
detecção de emoções por meio de sinais fisiológicos.

\section{MÉTODO}

Nesta pesquisa foi realizada uma revisão bibliográfica sistemática (RBS), com base no método de Sampaio e Mancini (2007). As autoras descrevem o desenvolvimento da revisão bibliográfica sistemática em cinco etapas:

\section{Definir a pergunta;}

\section{Buscar a evidência;}

3. Revisar e selecionar os estudos;

4. Analisar a qualidade metodológica dos estudos;

5. Apresentar os resultados.

Seguindo o método proposto pelas autoras, foi definida a seguinte pergunta de pesquisa:

- Quais são os principais equipamentos de medição de sinais fisiológicos, não invasivos ou pouco invasivos, passíveis de serem utilizados na mensuração quantitativa do nível de satisfação de usuários em testes de usabilidade?

A busca das evidências se deu em duas das principais bases de dados internacionais: Web of Science e Scopus. Foram analisados todos os artigos provenientes das buscas com a string: "physiological signal" AND ("user experience" OR satisfaction).

Foi definido que somente seriam avaliados trabalhos nos idiomas Inglês, português e espanhol, devido à proficiência do pesquisador responsável pelas revisões. Também foi definido que seriam excluídos da RBS, artigos não encontrados nas plataformas: Capes, Google, Google Scholar ou que não fossem fornecidos pelos autores.

As buscas não foram limitadas por data de publicação, de modo a incluir todos os artigos publicados nas bases selecionadas.

Os artigos identificados nas bases de dados $16^{\circ}$ Ergodesign - Congresso Internacional de Ergonomia e Usabilidade de Interfaces Humano Tecnológica: Produto, Informações Ambientes Construídos e Transporte

$16^{\circ}$ USIHC - Congresso Internacional de Ergonomia e Usabilidade de Interfaces Humano Computador

CINAHPA | 2017 - Congresso Internacional de Ambientes Hipermídia para Aprendizagem. passaram por duas etapas de revisão para serem considerados relevantes para a pesquisa. A primeira etapa foi baseada na análise do título, palavras-chave e resumo dos artigos encontrados.

A segunda etapa se deu com a análise integral do texto dos artigos que foram selecionados na etapa anterior, sendo que o critério de seleção dos trabalhos foi o nível de contribuição para responder a pergunta de pesquisa proposta. Os trabalhos que não apresentaram contribuições relevantes, em relação à identificação de emoções por meio de sinais fisiológicos, foram excluídos da pesquisa.

Durante o desenvolvimento da segunda etapa de seleção dos artigos foram extraídas as contribuições relativas aos equipamentos de medição de sinais fisiológicos.

Os resultados desta pesquisa são apresentados no item quatro, mas também contribuíram para o desenvolvimento da fundamentação teórica sobre os sinais fisiológicos. A fundamentação teórica foi ainda complementada por uma busca exploratória da bibliografia, a fim de esclarecer pontos não trabalhados nos artigos.

\section{FUNDAMENTAÇÃO TEÓRICA}

Esta parte do trabalho visa esclarecer os principais termos utilizados no desenvolvimento da RBS, assim como abordar de maneira sucinta os equipamentos de medição de sinais fisiológicos presentes nos artigos estudados.

\subsection{1 SINAIS FISIOLÓGICOS}

Segundo Goulart (2015) os sinais fisiológicos têm origem no Sistema Nervoso Central (SNC) e no Sistema Nervoso Periférico (SNP), sendo que estes podem ser avaliados para estudar as emoções humanas. Estes sinais são caracterizados por alterações nas taxas respiratórias, frequência cardíaca, suor, temperatura da pele, sinais cerebrais entre outros.

Os sinais fisiológicos são utilizados por pesquisadores da área de psicofisiologia para
Realização:

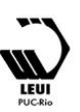




\section{$16^{\circ}$ \\ ERGODESIGN USIHC CINAHPA}

avaliar como estímulos psicológicos são refletidos no organismo humano, conforme definido por Andreassi (2001, p.35) em uma tradução livre:

"Psicofisiologia é o estudo das relações entre manipulações psicológicas e respostas fisiológicas resultantes, medidos no organismo vivo, para promover a compreensão da relação entre processos mentais e corporais."

\subsubsection{Ondas cerebrais - EEG (eletroencefalograma)}

Segundo Andreassi (2001, p.61), a primeira descrição de eletroencefalograma (EEG) ou "ondas cerebrais" aconteceu em 1875 pelo pesquisador Richard Caton. O pesquisador presenciou visualmente os efeitos que eletrodos posicionados diretamente no cérebro exposto, de coelhos e macacos, geravam no corpo destes animais quando aplicado uma corrente elétrica.

O funcionamento de um EEG se dá pela medição da tensão registrada entre seus eletrodos posicionados no couro cabeludo. Estes eletrodos são colocados em posições padronizadas distribuídas sobre a cabeça, e seu alinhamento geralmente é feito com uma touca com entradas para os eletrodos, como observado na Figura 1. Os eletrodos possuem códigos para indicar sua posição específica. Segundo o autor, os sinais neurais gravados com o equipamento são uma representação rudimentar, uma vez que, os eletrodos registram apenas o sinal perto da superfície do cérebro e por este motivo têm de ser filtrados antes da análise para não serem confundidos com outros sinais. (NACKE et al., 2010) $16^{\circ}$ Ergodesign - Congresso Internacional de Ergonomia e Usabilidade de Interfaces Humano Tecnológica: Produto, Informações Ambientes Construídos e Transporte

$16^{\circ}$ USIHC - Congresso Internacional de Ergonomia e Usabilidade de Interfaces Humano Computador

CINAHPA | 2017 - Congresso Internacional de Ambientes Hipermídia para Aprendizagem.

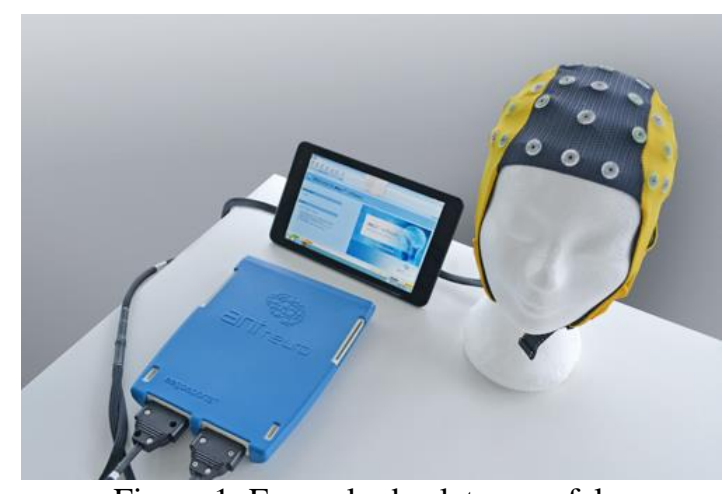

Figura 1: Exemplo de eletroencefalograma. Fonte: ANT Neuro (2016).

Goulart (2015) também afirma que pode haver ruídos nos sinais captados por um EEG, uma vez que, os sinais captados pelos eletrodos são de baixa amplitude e difíceis de adquirir. Isto se deve à dificuldade que os sinais têm de atravessar o couro cabeludo e o crânio, além de outras camadas.

\subsubsection{Condutividade da pele}

A condutividade da pele é medida através da resistência eléctrica proporcionada pela pele. Os sensores podem ser colocados nas palmas das mãos ou na sola dos pés, podendo indicar estímulos psicológicos mesmo quando não estão em contato direto com a superfície da pele.

Normalmente os sensores são posicionados em dois dedos da mão não dominante do indivíduo (LIU et al., 2014), conforme pode ser observado na Figura 2.

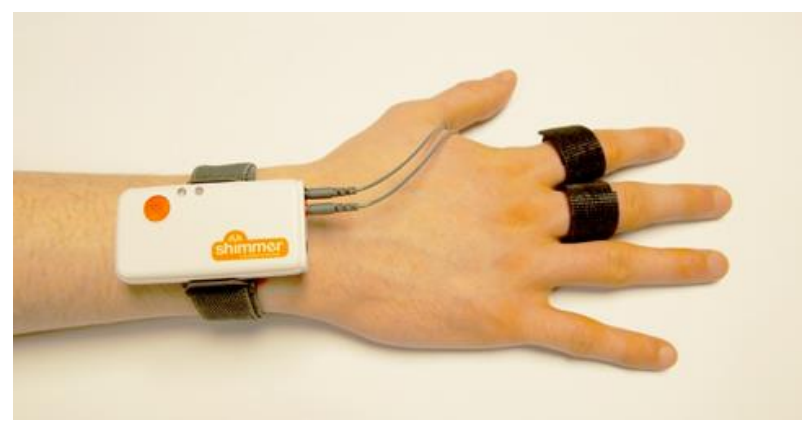

Figura 2: Exemplo de dispositivo para leitura da condutividade da pele. Fonte: Shimmer (2016).

Segundo Foglia e Zanda (2014), a condutividade da pele humana é afetada pela atividade do sistema nervoso simpático. Este sinal pode ser usado para 


\section{$16^{\circ}$ \\ ERGODESIGN USIHC CINAHPA}

indicar respostas emocionais de excitação, bem como de tensão e ansiedade.

\subsubsection{Frequência e pressão cardíaca}

Segundo Liapis et al.(2014), a frequência cardíaca é medida por sensores que captam a atividade elétrica do coração (Figura 3). A alteração dos batimentos cardíacos gerados pela atividade elétrica pode representar diferentes estados emocionais, como por exemplo, quando os batimentos cardíacos ficam acelerados é possível interpretar que o sujeito esta ansioso.

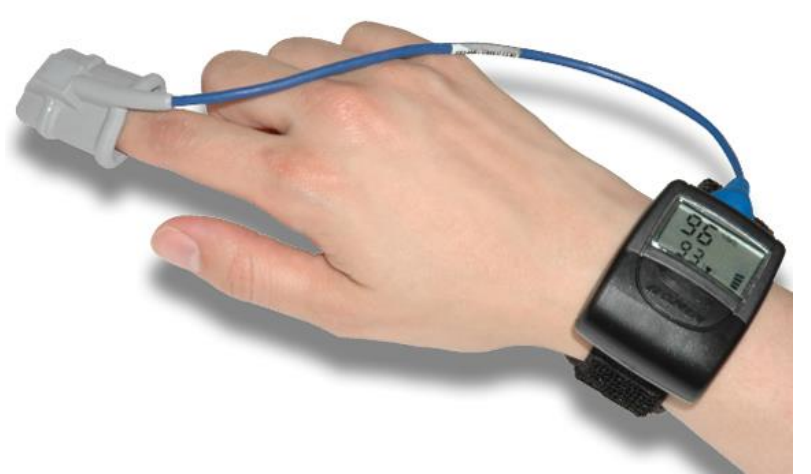

Figura 3: Exemplo de monitor de pressão e frequência cardíaca.

Fonte: Nonin (2016)

Segundo Foglia e Zanda (2014), o monitoramento da frequência cardíaca pode ser considerado uma boa forma de medir variações de estresse e relaxamento durante um experimento.

Segundo Andreassi (2001, p.386), a pressão cardíaca é influenciada pelos níveis de estresse, raiva e frustração do indivíduo. $\mathrm{O}$ autor afirma que um dos estados emocionais que pode ser mais bem observado através da pressão cardíaca é a raiva.

\subsubsection{Frequência respiratória}

A atividade nervosa simpática tem um efeito indireto sobre a frequência respiratória. As variações nas taxas de respiração podem indicar estados emocionais. O Ritmo de respiração acelerado pode indicar excitação por alegria ou raiva, enquanto a diminuição do ritmo pode indicar relaxamento e bem estar (FOGLIA e ZANDA, 2014; ZHOU et al., 2011). $16^{\circ}$ Ergodesign - Congresso Internacional de Ergonomia e Usabilidade de Interfaces Humano Tecnológica: Produto, Informações Ambientes Construídos e Transporte

$16^{\circ}$ USIHC - Congresso Internacional de Ergonomia e Usabilidade de Interfaces Humano Computador

CINAHPA | 2017 - Congresso Internacional de Ambientes Hipermídia para Aprendizagem.

\subsubsection{Detecção de expressões faciais}

No desenvolvimento do trabalho foram encontrados dois meios de detectar expressões faciais, a primeira usando eletromiografia (EMG) nos músculos da face (Figura 4) e a segunda por meio de softwares que traduzem as imagens captadas por uma câmera em expressões faciais (Figura 5).

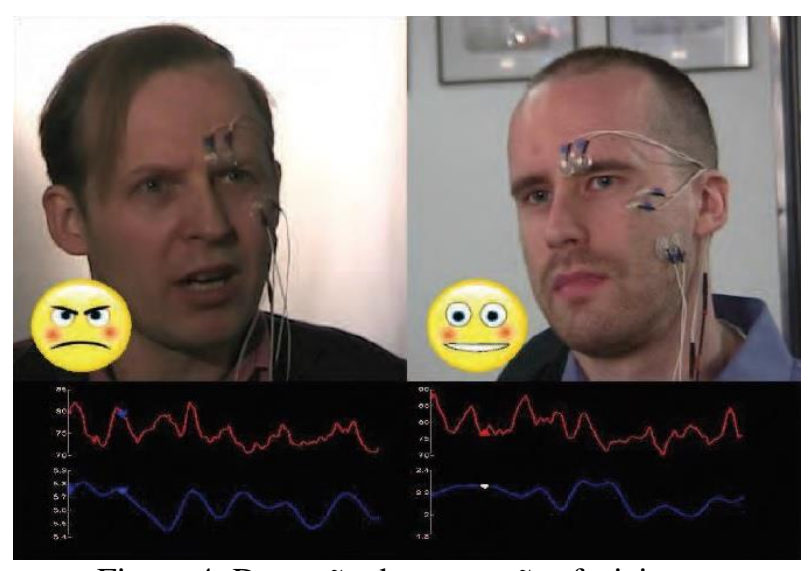

Figura 4: Detecção de expressões faciais por eletromiografia.

Fonte: Salminen et al. (2010).

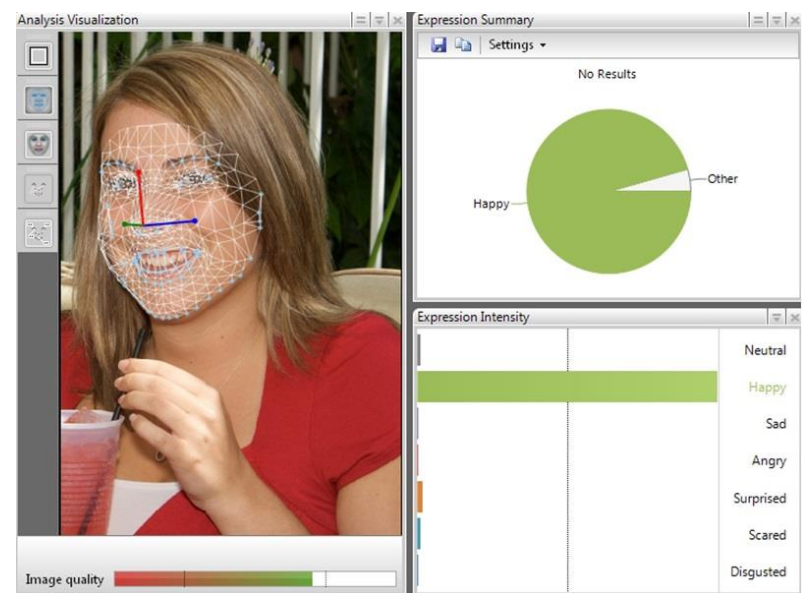

Figura 5: Detecção de expressões faciais por software. Fonte: De la Rosa Research (2016).

Segundo Zhou et al. (2011), o EMG mede a atividade muscular a partir da detecção e ampliação de impulsos elétricos gerados pela contração das fibras musculares. Os autores monitoraram o músculo zigomático maior e o músculo corrugador do supercílio no lado direito da face, para reconhecer as variações de estado 


\section{$16^{\circ}$ \\ ERGODESIGN USIHC CINAHPA}

emocional através de eletrodos EMG posicionados nestes músculos.

A detecção de expressões faciais por meio de software é descrita por Reale et al. (2013) como a identificação da face através de imagens em duas dimensões. O processo de identificação é iniciado pela determinação da posição da cabeça. $\mathrm{O}$ dimensionamento da face é baseado no centro dos globos oculares, a partir do qual é gerado uma malha, onde são analisadas as mudanças da face e descrito o rosto como um todo. Para melhorar o desempenho do software, o sistema salva expressões de cada usuário como modelos, que são utilizados pelo programa para comparar o estado atual do usuário com o banco de expressões armazenadas. Por meio de cálculos estatísticos de probabilidade são registrar as emoções atuais do usuário.

\section{EXPERIÊNCIA DO USUÁRIO MEDIDA POR MEIO DE SINAIS FISIOLÓGICOS}

As buscas por trabalhos científicos se deu conforme apresentado no método deste trabalho. $\mathrm{O}$ objetivo foi encontrar trabalhos com potencial relevância para o desenvolvimento de pesquisas que venham a trabalhar com a medição da satisfação do usuário, durante a interação com produtos, por meio de equipamentos de sinais fisiológicos.

No total foram encontrados 33 artigos nas bases de dados Scopus e Web of Science. Três destes artigos foram encontrados em ambas e um artigo estava disponível em idioma não incluído nas definições da pesquisa, restando 29 artigos para serem avaliados. Após a avaliação de títulos, palavras chave e resumos, foram filtrados 18 artigos com potencial relevância para a pesquisa. Após a leitura destes artigos, na íntegra, foram selecionados 10 artigos com relevância para o tema, conforme demonstrado na Figura 6. $16^{\circ}$ Ergodesign - Congresso Internacional de Ergonomia e Usabilidade de Interfaces Humano Tecnológica: Produto, Informações Ambientes Construídos e Transporte

$16^{\circ}$ USIHC - Congresso Internacional de Ergonomia e Usabilidade de Interfaces Humano Computador

CINAHPA | 2017 - Congresso Internacional de Ambientes Hipermídia para Aprendizagem.

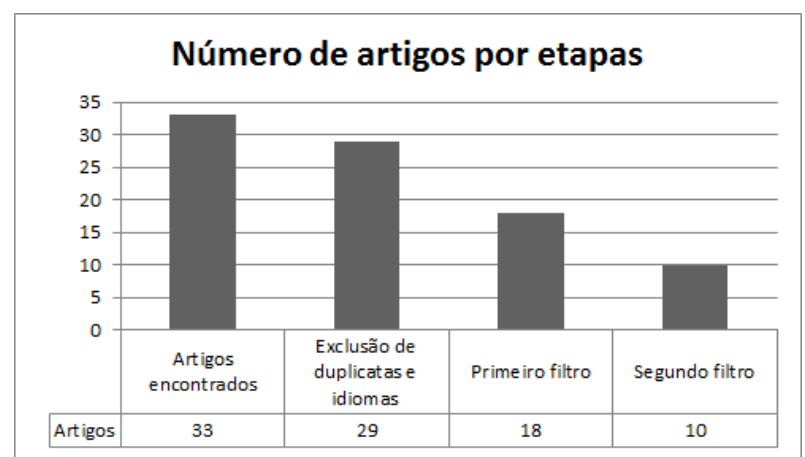

Figura 6: Número de artigos presentes em cada etapa da pesquisa.

Fonte: Os autores

As informações catalográficas, dos artigos selecionados, foram organizadas na Tabela $1 \mathrm{de}$ forma a permitir o desenvolvimento das análises bibliométricas.

Também é possível observar na tabela que quase todos os artigos tiveram no máximo cinco citações, sendo que três artigos não possuem nenhuma citação, com exceção do artigo de Nacke, et al. (2010) que obteve 49 citações. Este grande número de citações pode ser explicado pela consistência do estudo, devido ao grande aprofundamento nas análises estatísticas dos dados obtidos pelos pesquisadores. Os dados referentes ao número de citações foram obtidos por meio da base Google Scholar.

\begin{tabular}{|l|l|l|l|l|c|}
\hline Autores & Ano & Título & Fonte & País & Citações \\
\hline $\begin{array}{l}\text { Argyrio } \\
\text { u, V. et } \\
\text { al. }\end{array}$ & 2013 & $\begin{array}{l}\text { Guest editorial: } \\
\text { Introduction to } \\
\text { the special } \\
\text { issue on } \\
\text { modern control } \\
\text { for computer } \\
\text { games }\end{array}$ & $\begin{array}{l}\text { IEEE } \\
\text { transacti } \\
\text { ons on } \\
\text { cyberneti } \\
\text { cs }\end{array}$ & $\begin{array}{l}\text { Reino } \\
\text { Unido }\end{array}$ & 2 \\
\hline $\begin{array}{l}\text { Foglia, } \\
\text { P. and } \\
\begin{array}{l}\text { Zanda, } \\
\text { M. }\end{array}\end{array}$ & 2014 & $\begin{array}{l}\text { Towards } \\
\text { relating } \\
\text { physiological } \\
\text { signals to } \\
\text { usability } \\
\text { metrics: A case } \\
\text { study with a } \\
\text { web avatar }\end{array}$ & $\begin{array}{l}\text { Transacti } \\
\text { ons on } \\
\text { Compute } \\
\text { rs }\end{array}$ & Itália \\
\end{tabular}

Realização:

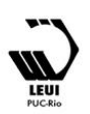


$16^{\circ}$ USIHC - Congresso Internacional de Ergonomia e Usabilidade de CINAHPA Interfaces Humano Computador

CINAHPA | 2017 - Congresso Internacional de Ambientes Hipermídia para Aprendizagem.

\begin{tabular}{|c|c|c|c|c|c|}
\hline $\begin{array}{l}\text { Liapis, } \\
\text { Alexand } \\
\text { ros and } \\
\text { Xenos, } \\
\text { Michalis }\end{array}$ & 2013 & $\begin{array}{l}\text { The } \\
\text { physiological } \\
\text { measurements } \\
\text { as a critical } \\
\text { indicator in } \\
\text { users' } \\
\text { experience } \\
\text { evaluation }\end{array}$ & $\begin{array}{l}\text { Proceedi } \\
\text { ngs of } \\
\text { the 17th } \\
\text { Panhelle } \\
\text { nic } \\
\text { Conferen } \\
\text { ce on } \\
\text { Informat } \\
\text { ics }\end{array}$ & Grécia & 2 \\
\hline $\begin{array}{l}\text { Liapis, } \\
\text { A. et al. }\end{array}$ & 2014 & $\begin{array}{l}\text { Evaluating } \\
\text { User's } \\
\text { Emotional } \\
\text { Experience in } \\
\text { HCI: The } \\
\text { PhysiOBS } \\
\text { Approach }\end{array}$ & $\begin{array}{l}\text { Lecture } \\
\text { Notes in } \\
\text { Compute } \\
\text { r Science }\end{array}$ & Grécia & 0 \\
\hline $\begin{array}{l}\text { Liu, Y. } \\
\text { et al. }\end{array}$ & 2014 & $\begin{array}{l}\text { A fuzzy } \\
\text { psycho- } \\
\text { physiological } \\
\text { approach to } \\
\text { enable the } \\
\text { understanding } \\
\text { of an } \\
\text { engineer's } \\
\text { affect status } \\
\text { during CAD } \\
\text { activities }\end{array}$ & $\begin{array}{l}\text { Compute } \\
\text { r-Aided } \\
\text { Design }\end{array}$ & $\begin{array}{l}\text { Reino } \\
\text { Unido }\end{array}$ & 5 \\
\hline $\begin{array}{l}\text { Nacke, } \\
\text { L. E. et } \\
\text { al. }\end{array}$ & 2010 & $\begin{array}{l}\text { Electroencepha } \\
\text { lographic } \\
\text { Assessment of } \\
\text { Player } \\
\text { Experience: A } \\
\text { Pilot Study in } \\
\text { Affective } \\
\text { Ludology }\end{array}$ & $\begin{array}{l}\text { Simulati } \\
\text { on \& } \\
\text { Gaming }\end{array}$ & Canada & 49 \\
\hline $\begin{array}{l}\text { Wobroc } \\
\text { k, D. et } \\
\text { al. }\end{array}$ & 2015 & $\begin{array}{l}\text { Continuous } \\
\text { Mental Effort } \\
\text { Evaluation } \\
\text { during 3D } \\
\text { Object } \\
\text { Manipulation } \\
\text { Tasks based on } \\
\text { Brain and } \\
\text { Physiological } \\
\text { Signals }\end{array}$ & $\begin{array}{l}\text { Human- } \\
\text { Compute } \\
\text { r } \\
\text { Interacti } \\
\text { on- } \\
\text { INTERA } \\
\text { CT 2015 }\end{array}$ & França & 3 \\
\hline $\begin{array}{l}\text { Zhou, F. } \\
\text { and } \\
\text { Jiao, R. } \\
\text { J. }\end{array}$ & 2013 & $\begin{array}{l}\text { Eliciting, } \\
\text { Measuring and } \\
\text { Predicting } \\
\text { Affect via } \\
\text { Physiological } \\
\text { Measures for } \\
\text { Emotional } \\
\text { Design }\end{array}$ & $\begin{array}{l}\text { Emotion } \\
\text { al } \\
\text { Engineer } \\
\text { ing vol. } 2\end{array}$ & $\begin{array}{l}\text { Estados } \\
\text { Unidos } \\
\text { da } \\
\text { Améric } \\
\text { a }\end{array}$ & 1 \\
\hline
\end{tabular}

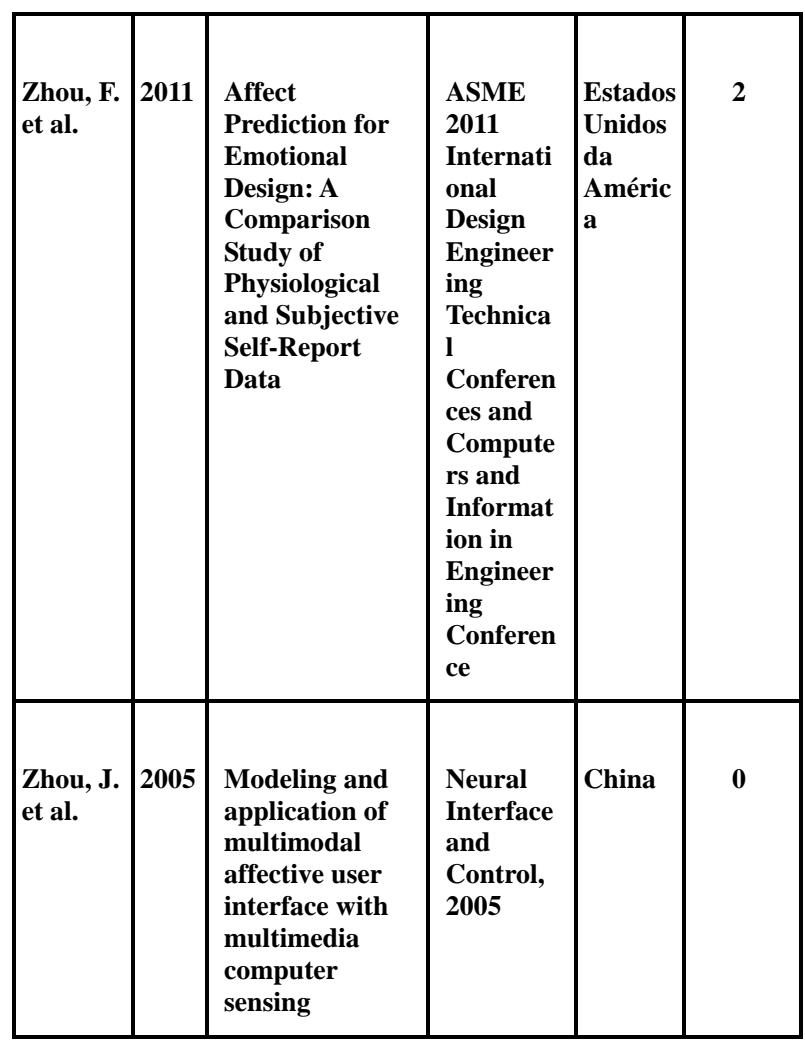

Tabela 1: Artigos selecionados Fonte: Os autores

É possível observar na Figura 7 que grande parte dos artigos provenientes das buscas sem filtro ficaram concentrados nos últimos oito anos, sendo o artigo mais antigo datado de 1973. Também é possível observar que os artigos selecionados após os filtros da RBS estão concentrados no período dos últimos cinco anos, e que o artigo mais antigo datado de 2005. Estes dados mostram que os pesquisadores buscavam maneiras de identificar emoções por meio de medições fisiológicas, utilizando equipamentos há mais de dez anos e que, ainda assim este é um campo que está em foco, possivelmente pela facilidade de acesso à novas tecnologias.

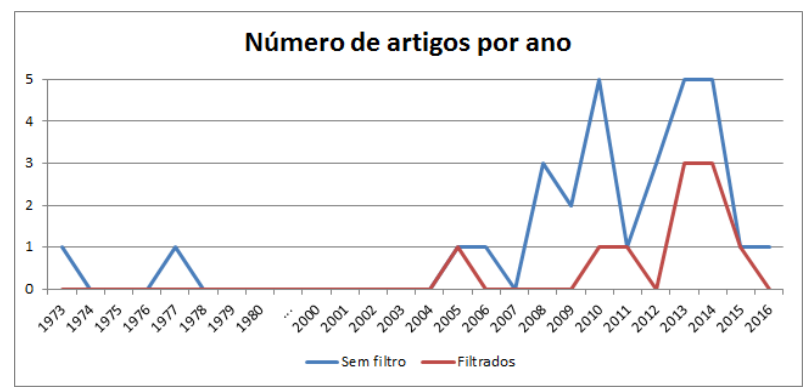




\section{$16^{\circ}$ \\ ERGODESIGN USIHC CINAHPA}

$16^{\circ}$ Ergodesign - Congresso Internacional de Ergonomia e Usabilidade de Interfaces Humano Tecnológica: Produto, Informações Ambientes Construídos e Transporte

$16^{\circ}$ USIHC - Congresso Internacional de Ergonomia e Usabilidade de Interfaces Humano Computador

CINAHPA | 2017 - Congresso Internacional de Ambientes Hipermídia para Aprendizagem.
Figura 7: Número de artigos por ano. Fonte: Os autores

A Tabela 2 contém o resumo de todos os artigos avaliados. Nota-se que metade dos estudos não só utiliza os equipamentos de medição fisiológica para aferir as emoções do usuário durante uma tarefa, como propõe uma nova forma de utilizar estes equipamentos. Foram propostos novos métodos de análise, assim como o desenvolvimento de sistemas que utilizam vários sinais fisiológicos para aferir de forma automática as emoções dos indivíduos testados.

\begin{tabular}{|c|c|c|}
\hline Autores & Ano & Resumo \\
\hline $\begin{array}{l}\text { Argyrio } \\
\text { u, V. et } \\
\text { al. }\end{array}$ & 2013 & $\begin{array}{l}\text { Editorial de uma edição focada em } \\
\text { equipamentos inovadores desenvolvidos para } \\
\text { jogos, sendo que um destes equipamentos } \\
\text { identifica as emoções dos usuários através do } \\
\text { reconhecimento das expressões faciais }\end{array}$ \\
\hline $\begin{array}{l}\text { Foglia, } \\
\text { P. and } \\
\text { Zanda, } \\
\text { M. }\end{array}$ & 2014 & $\begin{array}{l}\text { Desenvolve um teste de usabilidadeem dois } \\
\text { sistemas de marcação de exames on-line, para } \\
\text { aferir se a existência de um avatar têm impacto } \\
\text { positivo na resposta dos usuários. São utilizados } \\
\text { sinais fisiológicos para identificar o estado } \\
\text { emocional dos usuários enquanto participam do } \\
\text { teste. }\end{array}$ \\
\hline $\begin{array}{l}\text { Liapis, } \\
\text { Alexand } \\
\text { ros and } \\
\text { Xenos, } \\
\text { Michalis }\end{array}$ & 2013 & $\begin{array}{l}\text { Propõe uma nova abordagem para avaliar a } \\
\text { experiência emocional do usuário durante a } \\
\text { interação humano computador (HCI). Esta } \\
\text { abordagem utiliza sinais fisiológicos para gerar a } \\
\text { detecção automática das emoções do usuário } \\
\text { durante testes com o produto. Esta ferramenta } \\
\text { foi denominada PhysiOBS. }\end{array}$ \\
\hline $\begin{array}{l}\text { Liapis, } \\
\text { A. et al. }\end{array}$ & 2014 & $\begin{array}{l}\text { Propõe uma nova ferramenta para avaliar a } \\
\text { experiência emocional do usuário (user } \\
\text { emotional experience UEX) durante a interação } \\
\text { humano computador (HCI), utilizando sinais } \\
\text { fisiológicos em conjunto com questionários, } \\
\text { entrevistas e gravações do experimento. Esta } \\
\text { ferramenta foi denominada PhysiOBS. }\end{array}$ \\
\hline $\begin{array}{l}\text { Liu, Y. } \\
\text { et al. }\end{array}$ & 2014 & $\begin{array}{l}\text { O artigo propõe um método para avaliar os } \\
\text { aspectos emocionais envolvidos no } \\
\text { desenvolvimento de atividades usando } \\
\text { programas CAD, analisando seus operadores } \\
\text { durante a execução de tarefas. } O \text { método utiliza }\end{array}$ \\
\hline
\end{tabular}

\begin{tabular}{|l|l|l|}
\hline & & $\begin{array}{l}\text { três tipos de sinais fisiológicos, } \\
\text { elétroencefalografia, condutividade da pele e } \\
\text { eletrocardiografia. }\end{array}$ \\
\hline $\begin{array}{l}\text { Nacke, } \\
\text { L. E. et } \\
\text { al. }\end{array}$ & 2010 & $\begin{array}{l}\text { Apresenta um estudo piloto e os resultados } \\
\text { iniciais sobre a resposta emocional, medida } \\
\text { através de sinais fisiológicos, durante a interação } \\
\text { de jogadores com jogos virtuais. }\end{array}$ \\
\hline $\begin{array}{l}\text { Wobroc } \\
\text { k, D. et } \\
\text { al. }\end{array}$ & 2015 & $\begin{array}{l}\text { Estimação de carga de trabalho, através de } \\
\text { sinais fisiológicos (EEG, Condutividade da Pele e } \\
\text { Eletrocardiograma), na interação e } \\
\text { desenvolvimento de tarefas com objetos virtuais, } \\
\text { por meio do dispositivo CubTile em ambientes } \\
\text { virtuais }\end{array}$ \\
\hline $\begin{array}{l}\text { Zhou, F. } \\
\text { and } \\
\text { Jiao, R. } \\
\text { J. }\end{array}$ & 2013 & $\begin{array}{l}\text { Avalia o estado emocional de pessoas expostas a } \\
\text { estímulos visuais e auditivos, por meio de } \\
\text { dispositivos de leitura de sinais fisiológicos em } \\
\text { tempo real. }\end{array}$ \\
\hline $\begin{array}{l}\text { Zhou, J. } \\
\text { et al. }\end{array}$ & 2005 & $\begin{array}{l}\text { Apresenta o protótipo de um sistema de acesso a } \\
\text { emoções (MAUI) composto de sensores de sinais } \\
\text { fisiológicos, expressões faciais, sinais de voz e } \\
\text { dados comportamentais. }\end{array}$ \\
\hline $\begin{array}{l}\text { Zhou, F. } \\
\text { et al. }\end{array}$ & 2011 & $\begin{array}{l}\text { Compara a diferença da capacidade de } \\
\text { identificar emoções, a partir de imagens de seis } \\
\text { expressões faciais, por meio do relato dos } \\
\text { participantes e por leitura dos sinais fisiológicos } \\
\text { em tempo real. }\end{array}$ \\
\hline
\end{tabular}

Tabela 2: Resumo dos artigos selecionados. Fonte: Os autores

Na Tabela 3 são apresentados os equipamentos utilizados em cada um dos artigos estudados, bem como as emoções identificadas nos testes. Os equipamentos que apareceram com maior frequência, em mais da metade dos artigos, foram de condutividade da pele ( 7 artigos), frequência cardíaca (6 artigos) e eletroencefalografia (5 artigos). Esses equipamentos foram utilizados em um conjunto de no mínimo mais dois equipamentos de medição fisiológica, incluindo ou não condutividade da pele e frequência cardíaca, com exceção de um artigo, onde foi trabalhado exclusivamente a eletroencefalografia. A utilização de vários equipamentos pode ser explicada tanto pela presença de interferência nos sinais fisiológicos, como pela adequação do equipamento para com uma determinada emoção, como 


\section{$16^{\circ}$ \\ ERGODESIGN USIHC CINAHPA}

abordado anteriormente no referencial teórico. Desta forma se torna mais precisa a identificação de emoções em relação as alteração fisiológicas.

Este trabalho mostra um panorama geral dos equipamentos de medição fisiológica adotados para a identificação de estados emocionais de usuários no desenvolvimento de tarefas. É possível afirmar com base nos autores abordados que os sinais fisiológicos podem ser utilizados para a detecção de emoções positivas e negativas dos usuários, sendo que a diferenciação de emoções positiva e negativas entre si ainda não é precisa e requer maior desenvolvimento. Mesmo os métodos e equipamentos não apresentando distinção clara entre alguns estados emocionais, a visualização das alterações destes estados pode permitir que novas perspectivas sejam visualizadas e trabalhadas em testes de usabilidade.

Pela quantidade de artigos encontrados com a string de busca "physiological signal" AND ("user experience" OR satisfaction), no total de 33 artigos encontrados, que trabalha o tema de forma abrangente, é possível afirmar que ainda existem poucos estudos sobre o assunto. Isso demonstra uma possível oportunidade de pesquisa, tanto para as áreas de usabilidade e UX quanto para outras áreas onde as emoções do indivíduo sejam um fator relevante.

\section{CONCLUSÃO}

O desenvolvimento de ferramentas verbais e escritas que traduzem as emoções do usuário durante o desenvolvimento de testes de usabilidade é importante, porém, é possível que informações sejam suprimidas ou esquecidas pelos usuários durante o desenvolvimento dos testes de usabilidade. Estas ferramentas são importantes para aferir as impressões do usuário, mas como abordado no desenvolvimento da RBS, existem pesquisas que utilizam equipamentos de medição de sinais fisiológicos. Estes equipamentos podem preencher a lacuna de dados provenientes do momento em que o usuário interage com um produto ou sistema, enriquecendo os estudos na área de usabilidade e UX. $16^{\circ}$ Ergodesign - Congresso Internacional de Ergonomia e Usabilidade de Interfaces Humano Tecnológica: Produto, Informações Ambientes Construídos e Transporte

$16^{\circ}$ USIHC - Congresso Internacional de Ergonomia e Usabilidade de Interfaces Humano Computador

CINAHPA | 2017 - Congresso Internacional de Ambientes Hipermídia para Aprendizagem.

É possível afirmar que o objetivo do trabalho foi alcançado, por meio do método proposto de revisão bibliográfica sistemática. Foram identificadas e descritas as tecnologias presentes em todos os trabalhos selecionados, sendo estes analisados conforme proposto por Sampaio e Mancini (2007). Os artigos selecionados foram analisados e discutidos neste trabalho, assim como as 10 tecnologias abordadas nestes trabalhos. As tecnologias foram discutidas de maneira breve, de modo a permitir que novos pesquisadores entendam suas funções e possíveis usos em testes futuros.

Uma possível preocupação do uso de equipamentos de medição de sinais fisiológicos se relaciona com a naturalidade da interação do usuário com o produto, porém, novos equipamentos menos invasivos e de menor porte permitem que o usuário aja de maneira mais natural, interferindo minimamente nas atividades de interação com sistemas e produtos. Em contrapartida, estes equipamentos apresentam um alto custo, como no caso dos EEG mais sofisticados e precisos, o que pode dificultar o desenvolvimento de pesquisas com alguns dos equipamentos de leitura de sinais fisiológicos.

Mesmo com as dificuldades de acesso as tecnologias apresentadas, seu emprego na área de usabilidade e experiência do usuário pode ser considerado promissor. $\mathrm{O}$ uso de equipamentos de medição fisiológica nas áreas citadas pode levar a um novo nível de compreensão tanto das emoções dos usuários quanto de suas necessidades. Essas contribuições podem ir além da área acadêmica, auxiliando no desenvolvimento de produtos e sistemas que melhor se adequem as necessidades e capacidades de seus usuários.

\section{REFERÊNCIAS}

ALBERT, William; TULLIS, Thomas. Measuring the user experience: collecting, analyzing, and presenting usability metrics. Newnes, 2013.

ALEXANDROS, Liapis; MICHALIS, Xenos. The physiological measurements as a critical indicator 


\section{$16^{\circ}$ \\ ERGODESIGN USIHC CINAHPA}

$16^{\circ}$ Ergodesign - Congresso Internacional de Ergonomia e Usabilidade de Interfaces Humano Tecnológica: Produto, Informações Ambientes Construídos e Transporte

$16^{\circ}$ USIHC - Congresso Internacional de Ergonomia e Usabilidade de Interfaces Humano Computador

CINAHPA | 2017 - Congresso Internacional de Ambientes Hipermídia para Aprendizagem. in users' experience evaluation. Proceedings of the 17th Panhellenic Conference on Informatics. ACM, 2013. p. 258-263.

ANDREASSI, John L.; FILIPOVIC, Sasa R. Psychophysiology: Human Behavior and Physiological Response. Lawrence Erlbaum Associates, London. 2001.

ANT NEURO. Site do EEG Eeggosports, 2016. Disponível em: $<$ http://antneuro.com/news/correction-statement-publicationmentioning-eegosports>. Acesso em 06 ago. 2016.

ARAUJO, F. S. Avaliação da Experiência do Usuário: uma proposta de sistematização para o processo de desenvolvimento de produtos. Tese (Doutorado), Universidade Federal de Santa Catarina, Florianópolis. 2014.

ARGYRIOU, Vasileios et al. Guest editorial introduction to the special issue on modern control for computer games. Cybernetics, IEEE

Transactions on, v. 43, n. 6, p. 1516-1518, 2013.

\section{DE LA ROSA RESEARCH. Facereader por} software, 2016. Disponível em:

<http://www.delarosaresearch.com/detail.php?prod uctid=127\#description-tab>. Acesso em 06 ago. 2016.

DESMET, Pieter MA. Designing emotion. TU Delft, Delft University of Technology, 2002.

FOGLIA, Pierfrancesco; ZANDA, Michele; TRADING, I. O. N. Towards relating physiological signals to usability metrics: a case study with a web avatar. 2014.

GOULART, Christiane M. Uma contribuição ao estudo de sinais de EEG para avaliar estados emocionais e mentais de crianças com autismo na interação com robô móvel. Dissertação (Mestrado), Universidade Federal do Espírito Santo, Vitória, 2015.

HASSENZAHL, Marc. The effect of perceived hedonic quality on product appealingness.

International Journal of Human-Computer Interaction, v. 13, n. 4, p. 481-499, 2001.
HERTZUM, Morten; HOLMEGAARD, Kristin Due. Thinking aloud in the presence of interruptions and time constraints. International Journal of Human-Computer Interaction, v. 29, n. 5, p. 351-364, 2013.

JORDAN, Patrick W. An introduction to usability. CRC Press, 1998.

LAW, E.; HASSENZAHL, M.; ROTO, V.; VERMEEREN, A.; KORT, J. Understanding, scoping and defining user experience: a survey approach. Proceedings of the SIGCHI Conference on Human Factors in Computing Systems, CHI '09 Pages 719-728, ACM, 2009.

LIAPIS, Alexandros et al. Evaluating user's emotional experience in $\mathrm{HCI}$ : the physiOBS approach. Human-Computer Interaction. Advanced Interaction Modalities and Techniques. Springer International Publishing, 2014. p. 758767.

LIU, Ying et al. A fuzzy psycho-physiological approach to enable the understanding of an engineer's affect status during CAD activities. Computer-Aided Design, v. 54, p. 19-38, 2014.

NACKE, Lennart E.; STELLMACH, Sophie; LINDLEY, Craig A. Electroencephalographic assessment of player experience: A pilot study in affective ludology. Simulation \& Gaming, 2010.

NBR, ABNT. 9241-11. Requisitos ergonômicos para trabalho de escritório com computadores: Parte 11-Orientação sobre usabilidade. ASSOCIAÇÃO BRASILEIRA DE NORMAS TÉCNICAS. Rio de Janeiro: sn, p. 21, 2002.

NIELSEN, Jakob. Usability engineering. Elsevier, 1994.

NONIN. Site do dispositivo WristOx2, 2016.

Disponível em:

<http://www.nonin.com/WristOx3100>. Acesso em 06 ago. 2016.

REALE, Michael J. et al. Art critic: Multisignal vision and speech interaction system in a gaming context. Cybernetics, IEEE Transactions on, v. 


\section{$16^{\circ}$}

ERGODESIGN USIHC CINAHPA

$16^{\circ}$ Ergodesign - Congresso Internacional de Ergonomia e Usabilidade de Interfaces Humano Tecnológica: Produto, Informações Ambientes Construídos e Transporte

$16^{\circ}$ USIHC - Congresso Internacional de Ergonomia e Usabilidade de Interfaces Humano Computador

CINAHPA | 2017 - Congresso Internacional de Ambientes Hipermídia para Aprendizagem.

43, n. 6, p. 1546-1559, 2013.

ROGERS, Yvonne; SHARP, Helen; PREECE, Jennifer. Design de Interação. Bookman Editora, 2013.

RUBIN, Jeffrey; CHISNELL, Dana. Handbook of usability testing: how to plan, design and conduct effective tests. John Wiley \& Sons, 2008.

SALMINEN, Mikko et al. Psychophysiological Data Collection in an Organizational Setting:

Studying Interaction Between the Manager and Subordinate During Performance Review

Discussion. Measuring Behavior 2010, p. 55, 2010.

SAMPAIO, Rosana F.; MANCINI, Marisa C. Estudos de revisão sistemática: um guia para síntese criteriosa da evidência científica. Braz. J. Phys. Ther.(Impr.), v. 11, n. 1, p. 83-89, 2007.

SHIMMER. Site do dispositivo shimmer3 GSR + Unit, 2016. Disponível em:

<http://www.shimmersensing.com/shop/shimmer3wireless-gsr-sensor>. Acesso em 06 ago. 2016.

WHARTON, C. et al. The Cognitive Walkthrough: A Practioners Guide. Usability Inspection methods. p. 105-140. Wiley, 1994.

WOBROCK, Dennis et al. Continuous mental effort evaluation during $3 \mathrm{~d}$ object manipulation tasks based on brain and physiological signals.

Human-Computer Interaction-INTERACT 2015. Springer International Publishing, 2015. p. 472-487.

ZHOU, Feng; JIAO, Roger Jianxin. Eliciting, Measuring and Predicting Affect via Physiological Measures for Emotional Design. Emotional Engineering vol. 2. Springer London, 2013. p. 4162.

ZHOU, Feng et al. Affect Prediction for Emotional Design: A Comparison Study of Physiological and Subjective Self-Report Data. ASME 2011

International Design Engineering Technical Conferences and Computers and Information in Engineering Conference. American Society of
Mechanical Engineers, 2011. p. 917-925.

ZHOU, Jianzhong et al. Modeling and application of multimodal affective user interface with multimedia computer sensing. Neural Interface and Control, 2005. Proceedings. 2005 First International Conference on. IEEE, 2005. p. 28-31. 